\title{
Defining cancer-related fatigue for biomarker discovery
}

\author{
Kristin Filler $^{1} \cdot$ Leorey N. Saligan ${ }^{1}$
}

Received: 22 July 2015 / Accepted: 21 September 2015 / Published online: 5 October 2015

(C) Springer-Verlag Berlin Heidelberg (outside the USA) 2015

Cancer-related fatigue (CRF) is a prominent and distressing symptom for cancer patients that can impair their overall health-related quality of life [1]. CRF is often described as a complex and multidimensional concept consisting of physical, cognitive, affective, spiritual, psychosocial, and environmental factors $[1,2]$. Assessment of CRF is obtained from responses on various valid, self-report questionnaires established from several theoretical models of CRF. The complexity of CRF as a concept and the variability in the method of CRF assessment pose a challenge to investigators attempting to understand the etiology of CRF. Establishing a clear definition of a phenomenon, such as CRF, is an essential starting point for phenotypic characterization and biomarker discovery [3]. Therefore, this commentary aims to increase our awareness of the need for better definition and clearer phenotypic characterization of CRF.

Several organizations have proposed definitions for CRF such as the National Comprehensive Cancer Network (NCCN), the American Society of Clinical Oncology (ASCO), and the Pan Canadian Practice Guidelines [2, 4, 5]. However, a recent review conducted by the Multinational Association of Supportive Care in Cancer Fatigue Study GroupBiomarker Working Group found that CRF is defined in various ways [6]. Some studies referenced a specific definition used by national organizations such as the National Comprehensive Cancer Network [2], while other studies defined CRF using specific cancer treatment-related tools, such as the

Leorey N. Saligan

saliganl@mail.nih.gov

1 National Institute of Nursing Research, National Institutes of Health, 9000 Rockville Pike, Building 3, Room 5E, Bethesda, MD 20892, USA
National Cancer Institute Common Toxicity criteria, as well as diagnostic criteria from a proposed ICD-10 definition of CRF $[7,8]$. The lack of a consistent operational definition for $\mathrm{CRF}$ in the empirical literature makes it challenging to develop a CRF phenotype, which in turn poses a major obstacle for researchers investigating the biological mechanisms of CRF [9]. Therefore, consistent use of an agreed upon definition would be of benefit for biomarker discovery.

In addition to the variability in the definition of CRF, numerous questionnaires were reported in the reviewed articles to assess CRF. In fact, 23 different questionnaires were used to assess CRF in the 47 articles reviewed. Even when the same questionnaire was used, different scoring rubrics were often applied to phenotype CRF or to determine its clinical relevance $[10,11]$. The significance of this variability when investigating different biologic pathways to determine molecular correlates of CRF is pronounced. For example, we recently assessed genetic correlates of CRF using two different cut-off scores on the Functional Assessment of Cancer TherapyFatigue (FACT-F) questionnaire (http://www.facit.org/ FACITOrg/Questionnaires) in the same group of men with prostate cancer who received radiation therapy. The FACT-F subscale is a 13-item widely-used, readily available questionnaire validated to explore fatigue symptoms in the oncology population. Our unpublished findings revealed that different functional biologic pathways were found when different approaches were utilized to phenotype CRF patients using previously reported FACT-F cut-off scores (Fig. 1).

Investigating causal relationships of CRF with specific physiologic mechanisms will remain difficult, unless the complex concept of CRF is better defined, and clearly phenotyped. Perhaps, describing the disease and/or treatment associated with the CRF experience can better define the construct, especially if the intention is for biomarker discovery. CRF is often believed to be induced by the tumor itself and/or the 


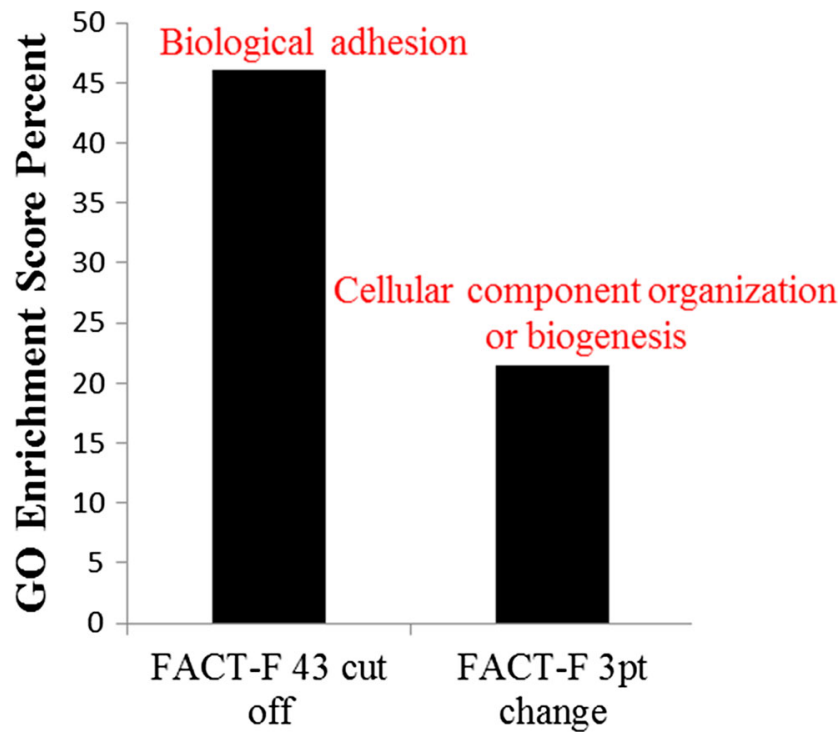

Fig. 1 This figure highlights the various biological pathways identified when different cut-off scores were applied to the Functional Assessment of Cancer Therapy-Fatigue (FACT-F) questionnaire. Gene ontology (GO) enrichment analysis is a way to assign significantly expressed genes into biologically meaningful GO categories; it is a method of biological interpretation. The higher the enrichment score, the more over-represented this functional group is in the gene list

mode of treatment [12]. Specifying the proposed inducer of CRF may place into perspective the physiologic mechanisms that may be involved. For example, a preclinical study demonstrated that an inflammatory response with corresponding fatiguing behavior was observed in a murine model of lung cancer while receiving a chemotherapeutic agent, etoposide; however, neither the behavioral or inflammatory responses were observed when using non-treated lung cancer mice [13]. In contrast, clinical studies have observed that some tumors (i.e., human papillomavirus (HPV)-related head and neck cancers) strongly induce pro-inflammatory responses [14]. Ionizing radiation, even partial body exposure, has been shown to induce pronounced neuroinflammation and consequent fatiguing behavior in a preclinical model [15]. Although more research is needed, there is also evidence that chemotherapy can influence pro-inflammatory mechanisms in preclinical and clinical models $[16,17]$. However, the evidence for fatigue mechanisms during concurrent chemoradiation therapy is not well developed. Therefore, the use of descriptive terms such as "radiation-related fatigue," "chemotherapyrelated fatigue," "tumor-related fatigue," or "survivorship" should be included along with descriptors of the clinical population being investigated to add perspective and context to the CRF definition, so a more precise CRF phenotype can be characterized.

Fatigue has been hypothesized to be influenced by several contributing factors, including stage of the disease, treatment status, or comorbid conditions [12]. For example, increases in inflammatory mechanisms have been observed in both acute and persistent fatigue related to cancer therapy; however, the mechanism of acute fatigue is often attributed to treatmentrelated anemia, whereas the mechanism of persistent fatigue following cancer therapy remains elusive $[1,18]$. In another study, it was observed that women with advanced stage ovarian cancer had higher inflammatory markers compared to women with early-stage disease [19].

Not only is the disease status of the clinical population important to describe when investigating CRF, it is also necessary to explain the effect of comorbid symptoms, such as depression and sleep disturbance. The occurrence of comorbid symptoms can complicate the identification of biological pathways of CRF. For example, depression is linked to increased inflammatory markers [20]. Depression often clusters with $\mathrm{CRF}$. The occurrence of depression and CRF together creates a more complicated mechanism in which to delineate distinct biological pathways for each symptom. Therefore, it is imperative to fully describe the population of interest, including the disease status and presence of comorbidities [9], in order to clearly define the CRF experience, characterize its clinicallyrelevant phenotype, and describe the biological mechanisms associated with the CRF phenotype.

In conclusion, in order to advance our understanding of the etiology of CRF, it is critical that we address the challenges mentioned in this commentary. An agreed upon operational definition of CRF, consistent use of an assessment tool and scoring criteria, and full descriptions of the context of CRF are necessary to move the CRF biomarker discovery forward. Operationally defining CRF will allow for optimal phenotypic characterization and will greatly enhance and advance the scientific base of CRF. Hence, specific terms such as "radiation-related fatigue," "chemotherapy-related fatigue," or "tumor-related fatigue" might be more useful when reporting biologic correlates or proposing etiologic mechanisms of CRF.

Conflict of interest The authors declared that they have no competing interests.

Financial disclosures This research was supported by the Division of Intramural Research, National Institute of Nursing Research, National Institutes of Health.

\section{References}

1. Bower JE (2007) Cancer-related fatigue: links with inflammation in cancer patients and survivors. Brain Behav Immun 21:863-871. doi:10.1016/j.bbi.2007.03.013

2. Kwak SM, Choi YS, Yoon HM, et al (2012) The relationship between interleukin-6, tumor necrosis factor- $\alpha$, and fatigue in terminally ill cancer patients. Palliat Med 26:275-282. doi:10. 1177/0269216311406991 
3. Mischak H, Allmaier G, Apweiler R, et al (2010) Recommendations for biomarker identification and qualification in clinical proteomics. Sci Transl Med 2:46 ps42. doi:10.1126/ scitranslmed.3001249

4. Bower JE, Bak K, Berger A, et al (2014) Screening, assessment, and management of fatigue in adult survivors of cancer: an American society of clinical oncology clinical practice guideline adaptation. J Clin Oncol 32:1840-1850. doi:10.1200/JCO.2013. 53.4495

5. Howell D, Keshavarz H, Broadfield L, et al. A pan canadian practice guideline for screening, assessment, and management of cancer-related fatigue in adults: Version 2-2015. Toronto: Canadian Partnership Against Cancer (Cancer Journey Advisory Group) and the Canadian Association of Psychosocial Oncology, April 2015. Retrieved from http://www.capo.ca/pdf/CRF Guideline.pdf

6. Saligan LN, Olson K, Filler K, et al (2015) The biology of cancerrelated fatigue: a review of the literature. Support Care Cancer 23: 2461-2478. doi:10.1007/s00520-015-2763-0

7. Alexander S, Minton O, Andrews P, Stone P (2009) A comparison of the characteristics of disease-free breast cancer survivors with or without cancer-related fatigue syndrome. Eur J Cancer 45:384-392. doi:10.1016/j.ejca.2008.09.010

8. Massacesi C, Terrazzino S, Marcucci F, et al (2006) Uridine diphosphate glucuronosyltransferase $1 \mathrm{~A} 1$ promoter polymorphism predicts the risk of gastrointestinal toxicity and fatigue induced by irinotecan-based chemotherapy. Cancer 106:1007-1016. doi:10. 1002/cncr.21722

9. Cashion A, Grady P (2015) The national institutes of health/ national institutes of nursing research intramural research program and the development of the national institutes of health symptom science model. Nurs Outlook 63:484-487. doi:10.1016/j.outlook. 2015.03.001

10. Wratten C, Kilmurray J, Nash S, Seldon M, Hamilton CS, O'Brien PC, Denham JW (2004) Fatigue during breast radiotherapy and its relationship to biological factors. Int J Radiat Oncol Biol Phys 59: 160-167. doi:10.1016/j.ijrobp.2003.10.008

11. Courtier N, Gambling T, Enright S, Barrett-Lee P, Abraham J, Mason MD (2013) Psychological and immunological characteristics of fatigued women undergoing radiotherapy for early-stage breast cancer. Support Care Cancer 21:173-181. doi: 10.1007/s00520-012-1508-6

12. Cheville A (2009) Cancer-related fatigue. Phys Med Rehabil Clin N Am 20:405-416. doi:10.1016/j.pmr.2008.12.005

13. Wood LJ, Nail LM, Perrin NA, Elsea CR, Fischer A, Druker BJ (2006) The cancer chemotherapy drug etoposide (VP-16) induces proinflammatory cytokine production and sickness behavior-like symptoms in a mouse model of cancer chemotherapy-related symptoms. Biol Res Nurs 8:57-169. doi:10.1177/1099800406290932

14. Tezal M, Scannapieco FA, Wactawski-Wende J, Hyland A, Marshall JR, Rigual NR, Stoler DL (2012) Local inflammation and human papillomavirus status of head and neck cancers. Arch Otolaryngol Head Neck Surg 138:669-675. doi:10.1001/archoto. 2012.873

15. Marquette C, Linard C, Galonnier M, Van Uye A, Mathieu J, Gourmelon P, Clarençon D (2003) IL-1beta, TNFalpha and IL-6 induction in the rat brain after partial-body irradiation: role of vagal afferents. Int J Radiat Biol 79:777-785. doi:10.1080/ 09553000310001610998

16. Seigers R, Schagen SB, Van Tellingen O, Dietrich J (2013) Chemotherapy-related cognitive dysfunction: current animal studies and future directions. Brain Imaging Behav 7:453-459. doi:10. 1007/s11682-013-9250-3

17. Bower JE, Lamkin DM (2013) Inflammation and cancer-related fatigue: mechanisms, contributing factors, and treatment implications. Brain Behav Immun 30:S48-S57. doi:10.1016/j.bbi.2012.06. 011

18. Ganz PA, Bower JE (2007) Cancer related fatigue: a focus on breast cancer and Hodgkin's disease survivors. Acta Oncol 46:474-479. doi:10.1080/02841860701367845

19. Lutgendorf SK, Weinrib AZ, Penedo F, et al (2008) Interleukin-6, cortisol, and depressive symptoms in ovarian cancer patients. J Clin Oncol 26:4820-4827. doi:10.1200/JCO.2007.14.1978

20. Raison CL, Capuron L, Miller AH (2006) Cytokines sing the blues: inflammation and the pathogenesis of depression. Trends Immunol 27:24-31. doi:10.1016/j.it.2005.11.006 